\title{
Individualisierung und Fiktionalisierung der Kann-Beschreibungen des Europäischen Referenzrahmens durch Dramapädagogik mit Handyfilmen
}

\author{
Ursula Christine Bünger
}

\begin{abstract}
Zusammenfassung
Ziel des vorliegenden Beitrags ist es, den Einsatz von dramapädagogischen und (handy)filmpädagogischen Elementen an die aktuelle fremdsprachendidaktische Diskussion in der Folge der Veröffentlichung des Europäischen Referenzrahmens und die reguläre curriculare Arbeit mit Nullanfängern anzubinden, gleichzeitig aber auch über die Grenzen des Referenzrahmens hinauszugehen und dafür anhand eines 25-stündigen dramapädagogischen Unterrichtsprojekts ein Beispiel zu liefern. In diesem Projekt wird die kognitive und pragmatische Enge der Prinzipien des Referenzrahmens (Europarat 2001) durch fiktionale und nicht-kognitive Elemente aufgelockert. Es stützt sich erstens auf das fremdsprachendidaktisch und szenisch aufgefasste Konzept der Interaktion sowie zweitens auf das Konzept des Kontexts des Sprachhandelns und -spielens. Affektive Lernerbedürfnisse werden darin mit kognitiven Zielen verbunden. Im Projektbericht wird besonders beleuchtet, wie die Lernenden selbst die im Unterricht entstandenen Kurzszenen mit ihrer Handyfilmkamera festhalten und ihre ,Handyfilme' zum Bezugspunkt für eine Auswertung der in einem solchen Unterricht gemachten Lernprozesse werden.
\end{abstract}

\section{Einleitung: Der Europäische Referenzrahmen und Dramapädagogik?}

Der vielerorts angesprochene ganzheitliche,Geist' der Dramapädagogik und die ausgesprochen pragmatische Ausrichtung des Europäischen Referenzrahmens (Europarat 2001; im Folgenden kurz als Referenzrahmen bezeichnet) scheinen einander zutiefst zu widersprechen (zur Kritik am Referenzrahmen, cf. Bausch et al. 2003). Schmenk hat dies schon 2004 in ihrem Aufsatz zum Zusammenhang zwischen Referenzrahmen und Dramapädagogik aufgezeigt: 
Language (in the Common European Framework for Languages, U.B.) is assumed to be a more or less neutral means of communication. [...] affective, intentional, intercultural etc. dimensions of language, communication and language learning are largely ignored (Schmenk 2004: 15)

Während Schmenk jedoch daraus den Schluss zieht, dass „drama elements appear to be barely compatible with the CEF“" (Schmenk 2004: 17), versuche ich in dem vorliegenden Aufsatz - obwohl ich sie teilweise auch kritisiere einige Prinzipien des Referenzrahmens dramapädagogisch zu realisieren. Ich bin damit also darum bemüht, einerseits den Anschluss an die Diskussion in der Sprachdidaktik um den Referenzrahmen zu finden und andererseits Dramapädagogik die sprachlichen Ziele des Referenzrahmens anzupassen. Mein hier dargestellter und begründeter Vorschlag lautet, kurze Szenen nach den Kann-Beschreibungen zu entwickeln, in einen fiktionalen Kontext zu situieren und abschließend mit den Handyfilmkameras der Lernenden zu filmen. Ich nutze dabei die schon im Referenzrahmen konzeptualisierte soziale Funktion von ,Sprache im Kontext', und bereichere sie um imaginäre bzw. fiktionale Elemente, und zwar unter Beibehaltung der Kann-Beschreibungen. Dies bezeichne ich als Prozess der Fiktionalisierung, sowie der der Individualisierung, da dieses Verfahren mir es möglich macht, aktuelle Lernerbedürfnisse und -ideen in den fremdsprachlichen Lehr- und Lernprozess mit einzubeziehen. Die Lernenden filmen abschließend mit ihren Handys die so entstandene interaktive Szene und sehen sich sofort danach selbst in der Fremdsprache sprechend und agierend wieder, welches meiner Erfahrung nach einen wichtigen Schritt zur Erlangung der Vertrautheit mit der Fremdsprache darstellt.

Zunächststelleich hier den Übergang von den Prinzipien des Referenzrahmens zu meinem dramapädagogischen Projekt dar, indem ich vor allem die darin enthaltenen KonzepteInteraktion und Kontext diskutiere. Außerdem gehe ich auf die Interaktionen mit dem Medium Handyfilmkamera ein. Achließend werde ich mein Vorgehen in einem 25-stündigen dramapädagogisch inszenierten Modul beschreiben.

\section{Anbindung an und Abgrenzung vom Referenzrahmen}

Zunächst möchte ich die für meine dramapädagogische Arbeit mit Handyfilmen bedeutenden Prinzipien des Referenzrahmens herausgreifen und auf mein Projekt beziehen.

\subsection{Ausrichtung auf Grammatik und Wortschatz}

Ein Blick auf die Inhaltsverzeichnisse in neuen Lehrwerken zeigt es: Die Unterrichtseinheiten sind (wieder) auf Grammatik und auf Wortschatz ausgerichtet. Ich begrüße die Ausrichtung auf den Wortschatz, da dies den Grundstein für mündliches Produzieren, Verstehen und Interagieren ist. Dem Comeback der 
Grammatik trage ich hier dadurch Rechnung, dass ich sprachlich korrekte Dialoge spielen lasse. Ich sehe also für die vorliegende Arbeit vom Kriterium der Verständlichkeit $\mathrm{ab}$, das für mich sonst bei mündlichen Interaktionen im Vordergrund steht.

\subsection{Zergliederung in Schritte und Kann-Beschreibungen: das Bausteinprinzip}

Die Lernschritte werden in den neuesten Lehrwerken nach dem modularen Prinzip in ,Häppchen' oder Module zerlegt. Diese Zerlegung führt dazu, Sprachenlernen als geradlinigen Prozess von Input (cf. Krashen 1985; Gass 1997) zu Output (cf. Swain 1985) zu verstehen, und nicht als spiralförmigen Prozess, der Elemente wieder aufnimmt, zusammenführt und erweitert. Trotz meiner Kritik daran habe ich in diesem Projekt ebenfalls sehr kurze Dialoge (,Häppchen') eingesetzt. Diese müssen jedoch selbstverständlich in einen curricularen Lernkontext eingebunden werden, der auch die Begegnung mit längeren Dialogen und Texten vorsieht.

\subsection{Lernerstrategien}

Schmenk (2004) kritisiert, dass der Strategienauffassung im Referenzrahmen einseitig das kognitive Modell des Kognitionspsychologen Anderson (2001) zugrunde liegt. Emotionale, imaginäre und körperliche Aspekte werden von diesem Ansatz ausgeschlossen.

Doch auch kognitive Aspekte sind Teil der Ganzheitlichkeit der Lernenden. Weiterhin bleibt das Ziel von Dramapädagogik im Fremdsprachenunterricht durchaus (kognitiver) Fremdsprachenerwerb. Dramapädagogische Elemente im Fremdsprachenunterricht erweitern jedoch die kognitiven und fremdsprachlichen Ziele des Referenzrahmens, indem spielerische und nicht-kognitive Elemente in den Fremdsprachenunterricht eingebunden werden.

In meinem Kurzprojekt habe ich weiterhin das (,unstrategische') Prinzip der Wiederholung und ,Einschleifung' durch Auswendiglernen eingesetzt, das manchmal in der Diskussion um Lernerstrategien unter den Tisch zu fallen scheint, aber auch eine zu trainierende Fähigkeit darstellt.

\subsection{Zielorientierung}

Die standardisierte Zielorientierung des Referenzrahmens (Krumm 2006: 31) läuft meines Erachtens Gefahr, die individuellen Lernerbedürfnisse und die konkreten Situationen und Lernkontexte aus den Augen zu verlieren. Ich habe dagegen versucht, Elemente aus der aktuellen Situation und den schulischen Raum in die Szenen mit einzubinden, die Ideen der Lernenden mit aufzunehmen und so eine Individualisierung vorzunehmen. Gleichzeitig habe ich mir für jede Szene als Ziel eine Kann-Beschreibung gesetzt und auch zu Anfang der Lerneinheit den Lernenden gegenüber so formuliert. Auf diese Weise 
habe ich versucht, konkrete Lernerbedürfnisse und standardisierte Lehrziele miteinander zu verbinden.

\subsection{Lernerautonomie}

Dievom Referenzrahmen (cf.Europarat 2001:40) angestrebte Lernerautonomie (cf. Martinez 2005: 66f) erfordert (meiner persönlichen Erfahrung im Rahmen des schulischen Fremdsprachenunterrichts nach) ein bestimmtes kognitives Niveau, sowie eine hohe Motivation - alles Variablen, die vielleicht eher im universitären Bereich gegeben sind. Ich sehe es dagegen (nicht nur) im schulischen Bereich als notwendig an, eine positive Beziehung nicht nur zwischen peers, sondern auch zwischen Lehrenden und Lernenden aufzubauen. Im Feld von nicht nur verbalen Interaktionen von Lehrenden und Lernenden kommt auch den Lehrenden eine nicht unerhebliche Rolle zu (cf. das Modell des teacherin-role in Schewe 1993), in der Form von der Förderung eines geschützten Lernklimas, Akzeptanz und Ermutigung von persönlichem Ausdruck. Ich sehe die Lehrperson also als mit-interagierenden Teil des lernenden Systems an.

\subsection{Projekt- und Produktorientierung}

Die Orientierung auf Endprodukte läuft manchmal Gefahr, die Prozesse des Lehrens und des Lernens aus den Augen zu verlieren. Ich vermute jedoch, dass durch das Sich-Selbst-Wiedersehen auf dem Bildschirm die Identität in der Fremdsprache gefestigt werden könnte. Die entstandenen Handyfilme haben außerdem eine dokumentarische Funktion für den Sprachlernprozess, und geben den Beteiligten das Gefühl, eine fremdsprachliche Leistung vollbracht zu haben.

\subsection{Einsatz digitaler Medien}

Zeitgleich mit der Einführung des Referenzrahmens werden Lehrende im Fremdsprachenunterricht in Europa dazu angehalten, unbedingt ,neue' Medien im Unterricht einzusetzen - als ob die bloße Anwesenheit von digitalen Medien unbedingt Unterricht verbessern könnte.

Trotzdem steht außer Frage, dass die Lernenden motiviert werden, wenn sie mit neuen Medien lernen. Aus diesem Grund habe ich den Handyfilm eingesetzt. Handys gehören in Italien noch mehr zum Alltag als internetgestützte Computer, und stellen also ein vertrautes, fast schon „privat“ zu nennendes Medium dar, das hier zum Lernmedium wird.

\section{Der Lernzielbereich Interaktion als Brücke zwischen Referenzrahmen und Dramapädagogik}

Nun möchte ich den Begriff der Interaktion als Verbindung zwischen Dramapädagogik und Referenzrahmen beschreiben, denn mit Interaktionen intendiere 
ich hier sowohl verbale als auch nicht-verbale Interaktionen. Das Konzept der Interaktion stellt so das Schlüsselkonzept zum vorliegenden Aufsatz dar, da im Weiteren auch die Interaktionen mit dem Medium beschrieben werden sollen. Die Interaktionen zwischen Lernenden und zwischen Lehrenden und Lernenden werden dagegen nicht eigens hervorgehoben werden.

Der Begriff der kommunikativen Aktivität unterscheidet im Referenzrahmen zwischen Produktion, Rezeption und Interaktion . Ziel von Dramapädagogik ist also nicht die „Fertigkeit“ Sprechen, sondern die Kategorie der mündlichen Interaktion. Krumm schreibt:

[D]ie Festlegung von „Interagieren“ als einem eigenen Lernzielbereich ist ein wichtiger (sprachdidaktischer; U.B.) Schritt. (Krumm 2008: 28)

Mündliche Interaktion unterscheidet sich also qualitativ von mündlicher Produktion (Sprechen) und mündlicher Rezeption (Hören). Während nun eine rein verbal verstandene Interaktion noch auf den Schulbänken sitzend stattfinden kann, reichert eine darüber hinaus non-verbal inszenierte Interaktion verbales Fremdsprachenlernen mit körperlichen, fiktionalen und emotionalen Elementen im ganzen schulischen Raum an. Ich verstehe Interaktion also nicht nur als verbal, sondern auch als non-verbal.

Während der Referenzrahmen weiterhin fast ausschließlich konkrete Interaktionen im Alltag und Berufsleben im Zielsprachenland im Sinn hat, schlage ich darüber hinaus unter dem Stichwort der fiktionalen Situierung vor, den Kann-Beschreibungen eine spielerische und fiktionale Richtung zu geben.

Zusammenfassend kann man also festhalten, dass sich dieKann-Beschreibungen des Referenzrahmens mittels der Erweiterung von einer verbal zu einer ganzheitlich verstandenen Interaktion mit dramapädagogischen Elementen verbinden lassen. Ein entscheidender Schritt ist dabei die Erweiterung der alltäglichen auf fiktionale Kontexte für die interaktiven Sprachhandlungen.

\section{Interaktionen innerhalb von fiktionalen Kontexten}

Alltagsauthentizität und Fiktionalität sind in der Fremdsprache stets an Kontexte gebunden. Sprachenlernen ist nicht nur ein kognitiver und psycholinguistischer, sondern auch ein sozialer Prozess. Die Kontextabhängigkeit von Fremdsprache ist ebenfalls ein Element, das ich dem Referenzrahmen (Europarat 2001: 140) entnehme (zum Fremdsprachenlernen als soziokulturellen Prozess, cf. Kramsch 1993 u.a.). Ich möchte hier also von Dramapädagogik als Interaktion im Kontext sprechen, und es so als fiktional situiertes Lernen (cf. Lave \& Wenger 1991) verstehen.

Der Europäische Referenzrahmen beschreibt jedoch als Kontexte der KannBeschreibungen (und somit von seiner Vorstellung von Fremdsprachenlernen) fast ausschließlich Kontexte von alltäglicher Lebensumwelt, Informationsaustausch, Mobilität und Berufspraxis (Europarat 2001: 59f). Diese Fokussierung auf alltägliche - Fremdsprache führt zu einer Unterbewertung der Fiktionalität im Fremdsprachenunterricht. 
Mein Vorschlag ist nun, die Kann-Beschreibungen in einen für die Lernenden fiktionalen bzw. ungewöhnlcihen Kontext zu situieren und dadurch zu fiktionalisieren. Die Lernenden stellen sich vor, in einem gemeinsam von Lehrperson und Lernenden erfundenen fiktionalen Kontext zu Theater zu spielen.

Dies geschieht durch die allmähliche Variierung der Kontexte, innerhalb derer die Kann-Beschreibungen sprachlich realisiert werden.

Ausgegangen bin ich zwar anfangs von Westermanns (2007) Vorschlag, für das Zielsprachenland authentische Situationen (ein Universitätscafé in Berlin) als Kontext für die interaktiven Szenen vorzuschlagen. Im Laufe des Projekts habe ich die Situierung jedoch variiert. Zunächst habe ich räumliche Kontexte der Schule selbst - Beispiele: Kaffeeautomat und Schulhofmauer - als für die Lernenden vertraute und somit für sie authentische Szenarien für die Kurzdialoge gewählt. Der Dialog auf der Schulhofmauer wurde dann verfremdet als in der Arztpraxis stattfindend durchgeführt. Danach habe ich eine ,Polizeiwache im Krimi' als aus dem Fernsehen vertrauten Kontext vorgeschlagen. Schließlich habe ich für meine Lernenden unvertraute und daher für sie fiktionale Kontexte, wie ein Beduinenzelt, als Orte des fremdsprachlichen Interagierens benutzt. Der unvertraute Kontext beflügelt die Phantasie der Lernenden und zum fiktionalen Interagieren.

Vertraute Kontexte führen $\mathrm{zu}$ alltäglichen und stereotypen verbalen und non-verbalen Interaktionen.

\section{Die zur Geste führende Funktion der Requisite}

Ich habe also durch die Umwandlung der alltäglichen in fiktionale Sprachspielkontexte die alltägliche Routine von verbalen Interaktionen „gestört“. Zur Veranschaulichung des Konzepts der Interaktion habe ich zu Beginn jeder interaktiven Kurzszene eine Geste mit einem realen, alltäglichen Objekt vorgeschlagen, das so zur Requisite wurde (beispielsweise einTaschentuch, eine Zigarette, ein Handy).

Diese Requisite bezog sich in meinen Szenen aber auf eine alltägliche Situation des Austauschens. Möglich wäre es aber auch, einen ,störenden Gegenstand einzuführen, der nicht zum Kontext passt (beispielsweise ein Brief in einer Situation zum Trinken statt der - tatsächlich eingesetzten - Coladose).

Die Requisite führt in der szenischen Arbeit zu einer Geste. Diese Geste trenne ich von der verbalen Interaktion. Daraus lernen die Lernenden, verbale Interaktionen in der Fremdsprache von nicht-verbalen Interaktionen zu unterscheiden. Dies sollte ihnen auch bewusst gemacht werden.

\subsection{Körperbewusstheit}

Die Einbeziehung der Geste in die Interaktionen führt nun zur Frage nach der Körperlichkeit in meinem Kurzprojekt. Als Input zur körperlichen Arbeit habe ich mich in diesem Projekt auf das Halten eines „gespannten Muskeltonus“ konzentriert. Dazu nutzte ich die Technik der Statuen nach Boal (cf. Schewe 
1993: 225ff.). Dabei „gefrieren“ die Schauspielenden in eine Geste und in eine Körperhaltung. Da es sich um szenische Interaktionen handelt, mache ich den Lernenden auch bewusst, dass Körperhaltung, Mimik und Gestik sich wie die verbalen Inhalte auf den Partner beziehen müssen. Ich fordere dazu auf, den Muskeltonus so anzuspannen, als ob der Körper mit elektrischer Energie geladen wäre. Zwischendurch bitte ich die Lernenden, sich zu entspannen und so den Unterschied zur Anspannung zu fühlen.

Diese zu haltende Spannung und Körperbewusstheit führt aus meiner Sicht zu erhöhter Konzentration und Aufmerksamkeit, und könnte zu dem kognitiven noticing fremdsprachlicher Strukturen führen, von dem Schmidt (1990: 129) spricht. ${ }^{1}$

\section{Fremdsprachenerwerb auch durch Wiederholung}

Damit habe ich schon die Frage nach dem Fremdsprachenerwerb bei dramapädagogischen Kurzsequenzen gestellt. Wettemann (2007) schlägt in seinem Aufsatz für den dramapädagogischen Unterricht mit Anfängern vor, nach der Output-Hypothese mit zunächst unanalysierten chunks zu arbeiten. Ich beziehe mich ebenfalls auf diese Output-Hypothese (cf. Swain 1985), die davon ausgeht, dass die Lernenden erst durch den Gebrauch von Sprache dazu gebracht werden, Fremdsprache auch formal zu verarbeiten. Da ich allerdings sehr viel weniger Zeit zur Verfügung hatte, habe ich Wettemanns Arbeitsweise etwas variiert. Ziel war für mich nur die Automatisierung und die Sprechflüssigkeit, die nach Wettemann nachfolgenden Schritte der Analyse und der Integration wurden von mir in der ersten, fremdsprachlich fokussierten Phase der Lerneinheit, sowie von einer Kollegin in einer gesonderten Lerneinheit nach dem Handyfilmprojekt durchgeführt.

Die Situation des Probens und Wieder-Probens und Einstudierens der Rollen, und zwar von sprachlichen und nichtsprachlichen Elementen, stellt für die Kunstform Theater eine Notwendigkeit dar. Das im Unterricht notwendige Einstudieren vor und während der Aufnahmen des Mini-Dialogs, so lange bis die Szene ,sitzt', gibt unmerklich Gelegenheit, fremdsprachliche Strukturen zu wiederholen und einzuschleifen.

Ich möchte also dafür plädieren, die unter dem Vorwand des Theater-Probens und Filmens notwendigen Wiederholungen nicht als Übel, sondern als Chance zu einem vertieften Behalten zu verstehen. Diese Vorbereitungen versorgen weiterhin die Lernenden mit Hör- und Sprechmaterial, fremdsprachliches Input (cf. Krashen 1985; Gass 1997) und Output (cf. Swain 1985), und zwar für Zuschauer und Schauspielende. Die Lernenden hoben gerade diesen Aspekt im Feedback als positiv für diese Art des Fremdsprachenerwerbs hervor. Das Entscheidende ist meiner Ansicht nach, dass die Lernenden die Wiederholung nicht als Wiederholung (typisch für die Institution Schule) wahrnehmen, sondern

\footnotetext{
${ }^{1}$ Cf. Eckert \& Riemer (2000), die in ihrem Aufsatz ,noticing' als Bindeglied zwischen emotionalem und kognitivem Fremdsprachenlernen beschreiben.
} 
als Spielen und für Theaterspielen notwendiges Proben. Weitere Lernzielbereiche sind Grammatik, Aussprache, Intonation und Sprechflüssigkeit. Aus affektiver Sicht führen die interaktiven Szenen nach Meinung der Lernenden zum relaxten Sprechen - ein Teilerfolg für Nullanfänger in der generell als „schwierig“ eingestuften Fremdsprache Deutsch. Ein weiterer Lernzielbereich, der auf diese Weise trainiert wird, ist nicht nur der grammatische (da es sich um formal korrekte Dialoge handelte), sondern auch der Bereiech der Aussprache, Intonation und Sprechflüssigkeit.

\section{Die Interaktionen mit dem Medium Handyfilmkamera}

McLuhan (1964) hat die Auswirkungen des Mediums auf die Botschaft bzw. auf den Medieninhalt postuliert. Ich gehe hier davon aus, dass das Medium Auswirkungen auf die Nutzer hat, dass aber auch die Nutzer das Medium aktiv benutzen können. Im Fall des Filmens mit der Handyfilmkamera von dramapädagogisch entwickelten Szenen bestimmen die Lernenden selbst die ,Einstellung(en)', die sie einnehmen möchten, werden aber auch vom Medium bestimmt. Das Handy wird im alltäglichen Gebrauch von den Lernenden meist zur Kommunikation eingesetzt. Beim Filmen von fremdsprachlichen, in Szene gesetzten Interaktionen wird aus dem alltäglichen Kommunikationsmedium jedoch ein Lernmedium. Wie wird nun beim Filmen von Szenen Fremdsprache gelernt? Im Folgenden möchte ich daher die Interaktionen der Fremdsprachenlernenden mit dem Medium Handyfilmkamera betrachten.

In der mir bekannten Literatur zu Dramapädagogik findet sich kein Hinweis auf die pädagogischen und fremdsprachdidaktischen Auswirkungen des Mediums selbst, was damit zusammenhängen mag, dass die Funktion des Kameramanns nicht wie in der vorliegenden Arbeit an die Lernenden übertragen wurde, sondern vom Kursleiter, von Kollegen oder Profis ausgeübt wurde. Grund für den Einsatz der Handyfilmkamera in der Hand der Lernenden war, dass mich die Lernerperspektive (im technischen wie im übertragenen Sinn) interessierte.

Weiterhin lag es in meiner Intention, dass die Lernenden selbst das Medium kontrollieren sollten. Denn nicht nur das Medium hat Auswirkungen auf den fremdsprachlichen und emotionalen Lernprozess, sondern die Lernenden sollen ein Stück weit lernen, ihrerseits mit dem Medium umzugehen.Videos wurden schon immer zur Dokumentation und (Selbst-)Evaluation (beispielsweise in Wettemann 2007) von Dramapädagogik eingesetzt. Kaum eine Arbeit befasst sich aber mit der Frage, welche kognitiven und affektiven Auswirkungen Videoaufnahmen auf die Theaterspielenden/Lernenden haben könnten, und schon gar nicht, was passiert, wenn nicht die Lehrperson oder Außenstehende, sondern die Lernenden selbst ihre Aufnahmen während ihrer szenischen Inszenierung in der Fremdsprache drehen. Mit der Handykamera ist jetzt ein den Lernenden vertrautes Medium auf dem Markt, mit dem die Lernenden nicht nur selbst aus verschiedenen Blickwinkeln (und zwar mehrere gleichzeitig) einfache Aufnahmen von ihren szenischen Interaktionen machen, sondern auch sich selbst sofort wieder sehen, einander und der Lehrperson zeigen 
und sich selbst und die anderen als Schauspieler und Fremdsprachenlernende evaluieren können. Somit werden die Lernenden nicht nur Protagonisten als verbale und nichtverbale Agierende bzw. Fremdsprachenspielende, sondern übernehmen auch die Kameraführung. Nicht zuletzt ,gehören' die Handyfilme den Lernenden und passen in jede Schul- und Handtasche. Damit können sich die Lernenden die Fremdsprache im wahrsten Sinne des Wortes aneignen.

Diese Interaktionen mit dem Medium können in die Interaktionen der Schauspielenden und Kameraführenden während des Aufnahmemodus und diejenigen während der Wiedergabe unterschieden werden.

\subsection{Aufnahmemodus}

Eine unmittelbare Wirkung des Mediums Handyfilm stellte die (handyspeicherkapazitätsbedingte) zeitliche Kürze der Dialoge dar. Fast alle Lernenden nehmen als Schauspielende vor der laufenden Handyfilmkamera spontan eine andere, „gespannte“, nicht alltägliche Körperhaltung ein. Als weitere Reaktion einiger Lernenden stellt sich in dieser Phase häufig Kichern ein, dem man mit Konzentrationsübungen begegnen kann.

Aus kognitiver Sicht stellt sich bei den Schauspielern das Bemühen ein, eine perfekte performance in der Fremdsprache zu liefern. In der Tat bewertete ich die Handyfilme als Leistungsnachweise in der Fremdsprache.

Das Gefilmtwerden kann jedoch auch zum Ablehnen des Gefilmtwerdens führen. Hier sollte die Lehrperson erspüren, ob es für die Lernenden einen Schritt in der persönlichen und fremdsprachlichen Entwicklung darstellt, sich vor einer Handyfilmkamera „zur Schau zu stellen“, oder ob die Ablehnung unbedingt respektiert werden sollte (ich halte es für eine positive Lernerfahrung, sich in der Fremdsprache sprechend gefilmt zu sehen).

Der Kameramann muss seine Aufmerksamkeit auf die zu filmenden Szenen fokussieren, befindet sich also vermutlich - wie Schauspielende und Zuschauende - ebenfalls in einem Zustand der ,gesteigerten Aufmerksamkeit“. Wird ein Dialogteil durch Zoomen hervorgehoben, wird dieser Zustand der gesteigerten Aufmerksamkeit noch erhöht, da sich der Kameramann sehr auf den fremdsprachlichen Text konzentrieren muss, um im geeigneten Moment den für das Zoomen gewählten Dialogteil hervorzuheben.

In einem ähnlichen Zustand der „gesteigerten Aufmerksamkeit“ befindet sich übrigens auch der Souffleur, der den Text zwar nicht auswendig vortragen muss, aber dem Dialog genau und aufmerksam folgen muss, um seine Aufgabe gut zu erfüllen. Ich bitte daher die Lernenden, die nicht gefilmt werden möchten, diese Aufgabe zu erfüllen.

\subsection{Wiedergabemodus}

Die Bemerkung von Wettemann (2007) zu den Reaktionen seiner Lernenden auf das Ansehen ,ihres' Videos führt mich zu der Fragestellung, die an dieser Stelle nicht beantwortet werden kann, welche evaluierende Wirkung das 
sofortige Sich-selbst-wieder-Sehen auf dem Handymonitor für Anfänger in der Fremdsprache hat. Ich vermute aus den Reaktionen meiner Lernenden, dass das szenische Sprachhandeln in der Fremdsprache zu einer Erfahrung der ,Differenz' bzw. der ,Andersheit' führt, die durch das Ansehen des Handyfilms gesteigert wird, denn meine Lernenden äußerten sich auf Nachfrage beim Wiederbetrachten der Filme dahingehend. Ich hoffe daher, dass das Sich-Sehen in der Fremdsprache zum Aufbau einer positiven Identität in der Fremdsprache führt. Beobachtet man die Lernenden, während sie sich selbst wieder sehen, kann man ebenfalls diesen Zustand der Spannung bemerken: keinem der Lernenden ist es gleichgültig, was da gerade auf den Handymonitoren passiert.

\section{Mein dramapädagogisches Unterrichtskurzprojekt}

Ich habe im ersten 25-stündigen Modul eines vom Europäischen Sozialfonds geförderten Deutschkurses an einem neusprachlichen Gymnasium in Süditalien mit Nullanfängern am Ende jeder 150minütigen Lerneinheit eine KannBeschreibung der Interaktion in Szene gesetzt und von einer oder mehreren Lernenden mit einer Handykamera aufnehmen lassen. Ich habe dabei die fremdsprachlichen Mittel bewusst sehr einfach gehalten.

Im institutionellen Kontext herrscht in Süditalien noch der Frontalunterricht vor. Allein die Tatsache, dass die Sitzordnung (Bankreihen vor dem Lehrertisch) durchbrochen wird, gibt den Lernenden ein Signal des ,Anderen'. Aus diesem Grund habe ich die Lernenden allmählich an die dramapädagogische Arbeit herangeführt.Bei der Lernergruppe handelte es sich um vierzehn 14-17-jährige Mädchen, von denen einige auch den Deutschunterricht am Vormittag besucht haben, darunter auch eine bilinguale Teilnehmerin. Ich habe die einzelnen Lerneinheiten in zwei Phasen geteilt: Während der ersten 90 Minuten vor einer Pause führte ich die fremdsprachliche Arbeit durch, bei der ich die für die interaktive Szene erforderlichen Dialogmodelle, Grammatik und Wortschatz einführte. Dabei formulierte ich für mich selbst und für meine Lernenden am Anfang der Stunde eine Kann-Beschreibung als Ziel. Die Stunde nach der Pause diente danach der Erarbeitung des Drehbuchs und der drama- und filmpädagogischen Arbeit.

\subsection{Vorgehen}

Zur fremdsprachlichen Arbeit: In den ersten 45 Minuten führte ich die fremdsprachliche Arbeit durch (Aufwärmen, Wortschatz, Grammatik, Fertigkeiten). Die Inhalte bauten progressiv aufeinander auf, wurden jedoch gleichzeitig modular als in sich geschlossene Einheiten geplant und durchgeführt. Dieses Vorgehen erwies sich deswegen als notwendig, da der Unterricht leider diskontinuierlich besucht wurde. Jeder Kann-Beschreibung habe ich im ersten Teil einen Modelldialog zugeordnet, den ich selbst verfasst hatte, und der während der fremdsprachlichen Arbeit zur Erarbeitung der grammatischen Regel diente, sowie im zweiten Teil einen szenischen Dialog, der gemeinsam 
mit den Lernenden erarbeitet und aufgeführt wurde. Beispielsweise fragte ich die Teilnehmer, mit welchem Transportmittel sie zur Schule kamen, während dagegen der aufgeführte szenische Dialog den Transport im All zum Thema hatte und an der NASA-Station situiert wurde.

Die Kann-Beschreibungen, die ich mit meinen Nullanfängern durchgeführt habe, waren: sich vorstellen (Name, Herkunft, Wohnort, Hobby), Handynummern austauschen (Zahlen und zu speichernder Name), sich über gelernte und gesprochene Sprachen, Sprachlehrerin und Lieblingsfach austauschen, über Geschwister und eigene Haustiere sprechen, jemanden zum Essen und Trinken auffordern, über Transportmittel zur Schule sprechen können. Es handelt sich also um Kann-Beschreibungen aus dem Lebens- und Schulalltag meiner Lernenden.

Wie oben beschrieben, habe ich diese Kann-Beschreibungen nach und nach in fiktiven Kontexten angesiedelt. Aus den Dialogen (im Anhang) ist ersichtlich, wie ich die Kontexte und damit die Dialoge im Lauf des Projekts verändert habe. Während die ersten Dialoge noch Alltagskontexten zugeordnet werden können, handelt es sich bei den letzteren um fiktionale Kontexte. Die szenische und filmpädagogische Arbeit, sowie der Prozess der Situierung wird nun genauer dargestellt.

Die szenische Arbeit begann nach einer zehnminütigen Pause. In der Pause schrieb ich den Anfang eines Modelldialogs an die Tafel und auf Rollenkärtchen, derdieKann-Beschreibung umsetzte, zur Situation passte und schnell auswendig gelernt werden konnte. Pro Situation schlug ich dazu einen passenden chunk vor. Beispielsweise habe ich den Imperativ („Nimm!“) nicht explizit grammatisch erarbeitet, sondern während dem szenisch dargestellten Geben und Nehmen eines Taschentuchs, also während der einleitenden Interaktion mit Requisit und Geste, unanalysiert vorgegeben.

\subsection{Szenische Arbeit}

Szenische Phase Eins: Heranführung an fiktionale Kontexte: Zuerst diskutierte ich mit den Lernenden den Kontext für die interaktive Szene. Ich schlage dazu Kontexte vor, greife aber Ideen der Lernenden und spontan entstehende Situationen auf.

Der erste Dialog (sich vorstellen) wurde szenisch nicht weiter ausgearbeitet, er diente dem gegenseitigen Kennenlernen, da die Lernenden aus verschiedenen Lerngruppen stammten. Er wurde an einer Schulbank vor der Tafel gespielt, wo so eine kleine ,Bühne' improvisiert worden war. Grund dafür war, dass sich die Lernenden an die neue Methode ,außerhalb der Schulbänke' gewöhnen sollten. Meiner Erfahrung nach ist es problematisch, wenn ich als Lehrende aus heiterem Himmel heraus ,neue und ,deutsche' Methoden in den Unterricht bringe; ich ziehe es vor, Veränderungen Schritt für Schritt vorzunehmen, denn ich halte es für wichtig, gewohnte schulische Abläufe nicht sofort, sondern allmählich zu verändern. Der zweite Dialog wurde am Kaffeeautomaten der Schule gespielt. Dieser Dialog spielt also in einem für die Lernenden alltäglichen 
schulischen Kontext außerhalb des Klassenzimmers.

Hier hatten die Lernenden auch die Idee, ,Statisten' zu engagieren: einige Lernende liefen durch die Szene, ohne zu sprechen. Während der Proben fordere ich die Lernenden (Zuschauer, Souffleur, Kameramännner und Schauspieler) auf, aktiv Ideen einzubringen, die ich, wie auch spontane Vorschläge, in das "Drehbuchëinarbeite.

Der dritte Dialog weist eine Mischform auf, denn zwar führt er auf verbaler Ebene ebenfalls eine alltägliche Kann-Beschreibung aus, er wurde aber in der Intonation verfremdet. Die Kann-Beschreibung des Dialogs ist der Austausch über gelernte Sprachen, Lieblingssprachen und Sprachlehrer, der Untertext aber und die nonverbale Szene bezog sich auf eine spontan entstandene Situation im Klassenzimmer. Diese Technik des „verfremdenden“ Untertextes (nach Stanislavskij) habe ich dabei so variiert, dass ich die Lernenden bat, sich den Kurzdialog als in einem anderen Kontext situiert vorzustellen. Ich griff die Tatsache auf, dass an diesem Nachmittag eine Lernende mit verletztem Knie in den Unterricht gehinkt war, und ließ sie diesen Dialog, der auf verbaler Ebene das Thema Lernen von Sprachen aufweist, als einen Dialog auf der Ebene der Intonation zwischen fürsorglichem Arzt mit verletztem Patienten und tröstender Mutter rezitieren.

Die weiteren drei Dialoge wurden alle in einem fiktionalen Kontext situiert (Krimi auf der Polizeiwache - im Beduinenzelt - an der NASA-Station).

Ich schlug dabei den Lernenden für die letzten drei Dialoge authentische und reale, aber vom eigenen Kontext entfernte Situationen vor.

Szenische Phase Zwei: Requisite und Geste als nonverbale Interaktion im Kontext: Für jeden Dialog habe ich weiterhin eine zufällig im Raum vorhandene Requisite eingesetzt. Bei dieser Requisite handelte es sich im zweiten Dialog um das Handy selbst, im dritten Dialog um eine Zigarette, im fünften Dialog um ein Taschentuch und im sechsten Dialog um einen Bleistift. Ziel war es, Interaktionen nicht nur verbal und non-verbal auszuführen, sondern auch einen realen Gegenstand ins Spiel zu bringen.

Die Requisite wurde an eine Geste (meist das Austauschen eines Gegenstands, wie beispielsweise einer Zigarette, da ich eine Schülerin mit ihren Zigaretten gesehen hatte) und an eine aus Frage / Aufforderung und Antwort bestehende fremdsprachliche Interaktion geknüpft. Damit konnte ich den Lernenden konkret verständlich machen, dass Körpersprache - in diesem Fall die Geste des Zigarettenanbietens (non-verbal) - und die verbale Interaktion in diesem ,Anfangsstadium der Schauspielerei ${ }^{`}$ zeitlich voneinander getrennt werden müssen. Damit vermittelte ich den Lernenden, dass Fremdsprachenlernen nicht nur eine rein verbale Interaktion ist und dass auch der Körper in solch einer banalen und alltäglichen Bewegung spricht. Konkret lernen die Lernenden hier, die Geste des Anbietens auszuführen, dann die Bewegung zu stoppen und die entsprechende Interaktion in der Fremdsprache zu verbalisieren. 
Szenische Phase Drei: Gemeinsame Verfassung des "Drehbuchs": Ich gab an der Tafel und auf den Rollenkärtchen den Anfang der Kurzszene vor, der die Kann-Beschreibung in Szene setzte, und ließ den Schluss von den Zuschauern und/oder von den Schauspielern mit erfinden. Ich erbat auch hier Änderungsund Erweiterungswünsche von den Lernenden und bezog sie mit ein. Ich schrieb während dieser Arbeit am Drehbuch kleine unanalysierte chunks, die im Gespräch entstanden, an die Tafel, während die Schauspieler selbst sie auf den Rollenkärtchen änderten.

Fremdsprachliche Zwischenphase: Sprechflüssigkeit, Aussprache und Intonation: Danach war es Aufgabe der Schauspielenden, den Kurzdialog zunächst von den Rollenkärtchen abzulesen und dann so weit wie möglich sofort auswendig zu lernen und flüssig zu sprechen. Dies gelang allen Lernenden, da ich die Dialoge extrem kurz hielt, um das Auswendiglernen zu erleichtern.

Da es sich um Nullanfänger handelte, habe ich einzelne Wörter und Sätze, die den Schauspielern besonders schwer fielen, während der Probe auch von den Zuschauern mit Fokussierung auf Aussprache und Intonation als unanalysierte chunks im Chor und einzeln wiederholen lassen. Die Herausforderung, fließend in der neuen Fremdsprache zu sprechen, lastete also nicht nur auf den Schauspielenden. Hilfreich ist es, wenn nicht nur der Lehrende und die jeweiligen Protagonisten, sondern beide Partner oder auch die ganze Lerngruppe den Text und die non-verbalen Elemente zusammen wiederholen, und einander vorsprechen oder vorspielen.

Mein Ziel ist also nicht Lernerautonomie, sondern dasjenige, ein Klima der gegenseitigen Unterstützung zwischen allen Beteiligten zu schaffen. Im Feedback meiner Lernenden tauchte dies als SSchaffen von Gruppensolidarität"wieder auf.

Szenische Phase Vier: Emotionale Färbung: Ich ließ den so entstandenen Dialog unter dem Vorwand des Auswendiglernens und des Aussprechens in verschiedenen emotionalen Färbungen sprechen. So eine Regieanweisung konnte lauten, den gesamten Dialog des Austauschs von Handynummern auf traurige Weise laut von den Rollenkärtchen abzulesen. Somit ließ ich verschiedene Emotionen ,ausprobieren'. Diese spielerische Aussprache löst Verkrampfungen, die sich beim Aussprechen der unbekannten Fremdsprache leicht einstellen.

Szenische Phase Fünf: Theaterprobe: Je zwei (einmal drei) Schauspieler gingen dann auf die im Klassenzimmer mit Schulbänken improvisierte Bühne (bzw. an den Kaffeeautomaten). Nicht nur ich, sondern auch die Zuschauenden gaben Feedback und Anregungen und wurden so mit in den szenischen Lernprozess einbezogen. Eine Lernende übernahm die Rolle der Souffleuse, kümmerte sich also um den verbalen Part. Diese Lernende konnte den Dialog in ihrem Heft mit- und bei Bedarf laut vorlesen.

Sobald die Schauspieler die Flüssigkeit in der Fremdsprache erreicht und den Text einigermaßen auswendig gelernt hatten, begann ich mit der eher 
theaterpädagogischen Arbeit an der Figur. Während der insgesamt neun Theaterproben habe ich folgende theatertechnische Elemente eingeführt: Rhythmus von verbalen und nicht-verbalen Elementen; zwischen den Sätzen eine Pause machen und atmen; sich Zeit lassen; Konzentration und Muskelspannung halten (dies gilt auch für die Zuschauenden!); Geste und Wort trennen (entweder man spricht oder man bewegt sich); Gesichtsausdruck; einen Satz sagen und dabei einen Untertext nach Stanislavskij denken bzw. emotional gefärbt sprechen.

\subsection{Handyfilmpädagogische Arbeit}

Sobald sich die Schauspieler sowohl im fremdsprachlichen Dialog als auch im schauspielerischen Ausdruck sicher fühlten und die Zuschauer auch fanden, dass die Szene filmreif war, wurde die Kurzszene mehrmals von einer oder mehreren Lernenden mit einer Handyfilmkamera aufgenommen.

Filmische Phase Eins: Flüssigkeit der Szene: Die ersten paar Male des Drehens und das erste Interagieren vor der Handyfilmkamera dienten der Gewöhnung an die Kamera und hatten als Ziele die flüssige Wiedergabe des Dialogs in der Fremdsprache ohne Stocken und zumindest ansatzweise mit dramatischem Ausdruck.

Von Anfang an trainierte ich die Lernenden darin, dabei einen imaginären Punkt hinter der Wand zu fixieren, um die Interaktion mit dem Medium in Gang zu bringen. Laut meiner Lernenden half ihnen vor allem dieser Hinweis, sich beim Gefilmtwerden zu konzentrieren und nicht in Kichern auszubrechen.

Meiner Erfahrung nach klappt dieses gefilmte Spielen nie im ersten Anlauf, sondern muss mehrere Male wiederholt werden. Ich möchte hier daran erinnern, dass es sich um Nullanfänger handelt, die einen Dialog auswendig lernen mussten, den sie teilweise noch nicht ganz analysiert hatten. Ich nehme also an, dass manchmal der pure Klang der Fragen und Antworten wiederholt wurde.

Filmische Phase Zwei: Hervorheben eines Frage/Antwortpaars durch Zoomen: Der Kameramann, die Schauspieler und die Zuschauer einigten sich auf ein Frage/Antwortpaar der Szene, das inhaltlich so bedeutungsvoll erschien, dass es durch Zoomen mit der Kamera oder durch Vorgehen hervorgehoben werden sollte. Dieser Ausschnitt aus der Szene durch Zoomen gab dem Kurzfilm seine ganz eigene Bedeutung (und sollte dann hoffentlich - aus fremdsprachlicher Sicht - besonders bemerkt und gelernt werden). Damit habe ich einen ersten Schritt hin zur Arbeit an der Perspektive und dem Filmen als aktiven Prozess hin in Richtung Kino (und nicht Filmen als das Nutzen eines passiven Instruments) gemacht.

Weiterhin ermunterte ich die Lernenden, beim Aufnehmen verschiedene Einstellungen im Raum vorzunehmen, erreichte also damit, dass wirklich mehrere Perspektiven von derselben Szene vorlagen. 
Filmische Phase Drei: Gemeinsames Ansehen der Filme: Nach den Filmen sahen sich die Lernenden gemeinsam mit mir den Handyfilm bzw. die Handyfilme auf den Monitoren an. Ich erbat dabei Feedback einerseits zur Drehtechnik, andererseits zum „Sich-selbst-in-der-Fremdsprache-spielen-Sehen“. Meistens bekam ich zur Antwort, dass sie sich „komisch“ fühlten. Andere Lernende reagierten mit Indifferenz. Diese Phase der gegenseitigen Evaluation und Selbstevaluation könnte mit Hilfe der Vorführung des Handyfilms mit einem Laptop, das für die ganze Klasse sichtbar ist (die Handymonitore weisen eine relativ kleine Dimension auf), noch weiter vertieft werden und wurde nur beim zehnten Treffen unmittelbar nach der Leistungsbewertung ausgeführt.

Einige der Lernenden berichteten, dass sie sich zu Hause den Handyfilm zum Vergnügen noch einmal angeschaut hatten. Diese Möglichkeit könnte sicher noch durch Aufgaben mehr ausgenutzt werden.

Die ersten Filme konnten im Computerlabor am Anfang der nächsten Stunde angesehen werden. Da dabei das Audio ausfiel, ließ ich die Lernenden die Dialoge live sprechen, während sie sich selbst auf dem Monitor spielen sahen.

Assimilation des Gelernten: Damit diese kurzen Dialoge nicht nur im Kurzzeitgedächtnis bleiben und zu einem gemeinsam erarbeitetem Drehbuch wurden, schrieb ich nach jeder Lerneinheit die Dialoge mit bzw. auf, tippte sie zu Hause ab und ließ sie von allen Lernenden in der nächsten Stunde als Warming-up und mit verteilten Rollen sprechen oder lesen (siehe oben).

Fremdsprachliche und szenische Zusammenführung: Leistungsbewertung und Feedback: Als Leistungsnachweis bat ich die Lernenden, für das letzte Treffen je einen Kurzdialog auswendig zu lernen. Während dem letzten Treffen wurde im Klassenzimmer (und am Kaffeeautomaten der Schule) die Bühne eingerichtet, und die Kurzszenen wurden alle noch einmal vorgespielt. Bewertet wurden die Flüssigkeit des Sprechens in der Fremdsprache und die Verständlichkeit.

Ich habe es dabei - in der Rolle sowohl der Fremdsprachenlehrenden als auch der der Dramapädagogin - für mich selbst schwierig gefunden, die schauspielerische Performanz getrennt von den fremdsprachlichen Fähigkeiten zu bewerten. Ich habe dieses Dilemma in diesem Projekt so gelöst, dass ich versucht habe, nur die fremdsprachliche Performanz zu bewerten, da es sich um Theater für den Fremdsprachenunterricht handelt.

Allen Lernenden gelang es, einen der Dialoge auswendig und flüssig zu spielen und zu sprechen, außer den schon erwähnten zwei Lernenden, die nicht Theater spielen wollten und ihren Dialog daher in der Schulbank sitzend sprachen. Damit hatten alle das Ziel des Fremdsprachenerwerbs durch das Handeln in fiktiven Kontexten erreicht. Meiner Erfahrung nach können fremdsprachliche (kognitive) Lernziele daher auch effektiv durch ganzheitliches und szenisches Lernen erreicht werden, da auf diese Weise zusätzlich affektive Lernerbedürfnisse beachtet werden. Fremdsprachliche Dialoge können also nicht nur durch Verstehen, metalinguistische Analyse und kognitive Strategien, sondern auch durch spielerische Wiederholung eingeprägt werden. Dieses 
Ergebnis finde ich vor allem im Hinblick auf Schulstufen wichtig, auf denen Lernende nicht unbedingt auf den Einsatz von kognitiven und metakognitiven Lernstrategien ansprechen.

Zur abschließenden Selbstbewertung stellte ich die Frage, was die Lernenden in diesem Projekt für die Fremdsprache und für „sich selbst“ gelernt hatten. Die Lernenden antworteten auf die erste Frage, dass sie es positiv fänden, nicht auf den Schulbänken sitzend zu lernen; dass sie Hören und Sprechen gelernt hätten; lockereres Sprechen; und dass die vielen Wiederholungen nützlich für das Einprägen ins Gedächtnis gewesen wären. Das Antworten auf die zweite Frage fiel den Lernenden sichtlich schwerer; es fielen nur Stichworte wie Stärkung der Gruppenzusammengehörigkeit und Gedächtnistraining, vielleicht ein Indiz dafür, dass schulisches (kognitives) und außerschulisches (nicht kognitives) Lernen als strikt getrennt gesehen werden.

Die Analyse und Integration der grammatischen Phänomene wurde in einem 25-stündigen Modul durchgeführt, das sich an dieses Theatermodul anschloss.

Filmische Endphase: Erstellung des Produkts DVD: Geplant war, dass die Lernenden ihre Handyfilme nach jeder Lerneinheit zu Hause auf ihren Computer überspielen, zippen und an die Emailadresse der Deutschlehrerin schicken sollten. Dies war mit Schwierigkeiten verbunden, da nicht alle Lernenden DSL-Internetanschluss hatten (was ich stillschweigend vorausgesetzt hatte).

Das Überspielen der Handyfilme auf einen Computer hätte auch einen Schritt in die Überwindung der - genderbedingten? (meine Lerngruppe bestand aus vierzehn Mädchen) - Scheu vor Technik sein sollen und können. Doch nur eine meiner Lernenden nutzte gekonnt die bluetooth-Technik ihres Handys, lud das Handyvideo auf ihren Computer, zippte die Dateien und schickte sie mir per Email. Daher war beim letzten Treffen ein Techniker anwesend, der alle technischen Fragen (beispielsweise zur Software) löste, selbst noch einmal alle Szenen mit seinem Handy während der Leistungsbewertung filmte, und den Lernenden auf seinem Laptop alle Szenen noch einmal vorspielte.

Die Szenen, die von mir und dem Techniker als am besten gelungen bewertet wurden, wurden in einem professionellen Studio mit Untertiteln versehen. Am Ende des Projekts wurden alle Handyfilme auf eine CD kopiert, mit einem Cover versehen und allen Lernenden für das Dossier ihres Sprachenportfolios zusammen mit dem Leistungsnachweis für das Modul ausgehändigt.

\section{Schlussfolgerungen, Grenzen und Ausblick}

Ich hoffe, dass ich mit meinem Projekt zeigen konnte, dass sich die KannBeschreibungen aus dem Referenzrahmen und Dramapädagogik nicht ausschließen müssen und dass dramapädagogische Elemente auch erfolgreich mit Nullanfängern innerhalb eines knapp bemessenen Zeitrahmens im schulischen Bereich eingesetzt werden können. Dadurch, dass ich den Begriff der Interaktion ernst genommen habe und den pragmatischen Begriff des Kontexts aus dem 
Referenzrahmen allmählich um eine fiktionale Komponente bereichert habe, konnte ich mit meinen Lernenden Kann-Beschreibungen auf spielerische und motivierende Art und Weise umsetzen. Weiterhin gab mir die Theater-Arbeit die Möglichkeit, Elemente in die Szene aufzunehmen, die sich aus der jeweiligen Situation ergaben. Die habe ich als Fiktionalisierung und Individualisierung der Prinzipien des Referenzrahmens bezeichnet.

Durch den Einsatz der Handyfilmkamera schließlich habe ich ein alltäglich gewordenes Kommunikationsmedium in ein Lernmedium umgewandelt. Auch hier habe ich mich auf die Interaktion zwischen Lernmedium und Lernenden sowohl auf Seite der Schauspieler („Blick auf einen Punkt hinter der Handykamera") als auch auf Seiten der Kameramänner (Zoomen) bezogen. Freilich konnte dies im vorgegebenen Zeitrahmen nur in Ansätzen geschehen.

\subsection{Grenzen des vorliegenden Kurzprojekts}

Ziel des Projekts war es also insgesamt, kognitive und fremdsprachliche (Referenzrahmen) und fiktionale, affektive und soziale Elemente (Dramapädagogik) des Fremdsprachenlernens zu integrieren. Es sind jedoch noch Studien notwendig, die den von mir postulierten Zusammenhang zwischen Körperspannung und Aufmerksamkeit wissenschaftlich nachprüfen. Fördert die gesteigerte Aufmerksamkeit der Lernenden in der Rolle der Zuschauer, Schauspieler, Souffleure und Kameramänner wirklich das Fremdsprachenlernen?

Das hier dargestellte Vorgehen gründet aus kognitiver Sicht auf die Nutzung der Wiederholung durch das Proben. Damit bekommen die Zuschauenden und Kameraführenden fremdsprachlichen - hoffentlich vergnüglichen - Input (cf. Krashen 1985), während die Schauspieler nach der Output-Theorie (cf. Swain 1985) die Fremdsprache lernen. Metakognitive Lernstrategien wurden nicht eingesetzt, auch die Zeit für die metalinguistische Analyse war knapp bemessen. Dafür habe ich das Prinzip der spielerischen Wiederholung gezielt eingesetzt. Es bleibt noch zu erforschen, inwieweit und ob dieses fremdsprachliche Lernen durch Wiederholung doch zu sehr an der Oberfläche bleibt.

Bedingt durch die (noch) begrenzte Speicherkapazität der Handys, die ,Kurzschrittigkeit' der Moduldidaktik, die Konzentration auf einzelne KannBeschreibungen, sowie die Rücksichtnahme darauf, dass die Schauspieler in einer für sie noch fremden Sprache Dialoge ad hoc auswendig lernen mussten, sind meine Dialoge von extremer Kürze. Die hier vorgestellten, nach der Output- Hypothese szenisch eingeübten Modelldialoge beruhen im Prinzip auf der kleinschrittig vorgehenden Kürze der Moduldidaktik, die im ersten Teil des Artikels kritisiert wurde. Diese Vorgehensweise muss natürlich im curricularen Fremdsprachenunterricht ergänzt werden. Somit stellt diese „Handyfilm-Dramapädagogik“ eine Möglichkeit dar, einen Teil des Curriculums motivierend zu gestalten.

Die Möglichkeit der sofortigen (Selbst-) Evaluation auf den Handybildschirmen, aber auch diejenige der (Selbst-) Evaluation durch Wiedergabe der Handyfilme auf einem Computerbildschirm könnte sicher auch noch ausgebaut 
werden. Es genügt also nicht, einfach den Handyfilm vorzuführen, es müssen auch entsprechende Fragen gestellt werden, die vom einfachen „Wie fühlst du dich, wenn du dich auf Deutsch spielen siehst?" bis hin zur Selbstkorrektur von fremdsprachlichen Fehlern und theatralischem Ausdruck reichen könnte.

Aus dramapädagogischer Perspektive ließen diese kurzen interaktiven Sequenzen aufgrund des Sprachstandes keine Entwicklung der Interaktion zu einem dramatischen Konflikt hin mit anschließender Lösung zu. Es ist also selbstkritisch zu reflektieren, dass es sich bei dem vorliegenden Projekt eher um ein überschaubares ,Interaktionsprojekt' als um ein komplexeres dramapädagogisches Projekt handelt. Womöglich wurde den Kann-Beschreibungen im Referenzrahmen das Dramatische am Theaterspiel im Fremdsprachenunterricht ,geopfert'. Dieser dramatische Aspekt könnte ebenfalls in einem Projekt, in dem mehr Zeit vorhanden ist, anders ausgearbeitet und betont werden.

Aufgrund der geringen fremdsprachlichen Vorkenntnisse der Lernenden übernahmen diese Rollen, die ihnen charakterlich zusagten. In längeren Projekten habe ich dagegen immer darauf zugearbeitet, dass auch dem eigenen Charakter entgegen gesetzte Rollen und Emotionen als Erweiterung der persönlichen Ausdrucksfähigkeit ausprobiert werden können. Viele Elemente, wie die Anfänge der Dialoge, sowie Vorschläge für Kontexte und Requisiten sind von mir als Lehrperson gekommen. Ich frage mich, ob man hier die Lernenden noch mehr mit einbeziehen könnte. Mit einer anderen dramapädagogischen Gruppe, in der ich weniger an der fremdsprachlichen Zielsetzung arbeitete, habe ich in der Tat bemerkt, dass viele Jugendliche Mühe haben, selbstständig und schnell fantasievolle Kontexte und Aktionen zu improvisieren. Trotzdem würde ich in einem Nachfolgeprojekt versuchen, mir als Ziel noch mehr die Entwicklung der Phantasie der Jugendlichen zu setzen..

Um die Erfahrungswelt der Lernenden noch mehr in das Projekt mit einzubeziehen und um die Assimilation des Gelernten („Was hast du heute gelernt?") zu fördern, wäre es vielleicht gut gewesen, eine kognitive Selbstbewertung und ein erfahrungsbezogenes Feedback nicht nur am Ende des Projekts, sondern am Ende jeder Lerneinheit durchzuführen. Was die technische Seite betrifft, hatten die Lernenden laut ihren eigenen Angaben keine Zeit oder kein ausreichendes technisches Know-How, um sich zu Hause an den Computer zu setzen, den Handyvideofilm darauf zu laden und mir den Handyfilm per Email zu schicken; außerdem kam es vor, dass ein Handy gerade nicht aufgeladen war; oder dass die Lernenden das Handy zu Hause vergessen hatten. Außerdem weisen Handyfilme noch keine hohe Bildqualität auf. So musste zur Produktion der DVD am Schluss doch ein Techniker eingesetzt werden Das (untergeordnete) medienpädagogische Ziel des souveränen Umgangs mit Handyfilmtechnik und Computer wurde also nicht von allen Teilnehmenden erreicht.

Ich selbst habe den (mir selbst auferlegten) Zwang zu einer gefilmten Szene am Ende jeder Lerneinheit bzw. zum Endprodukt manchmal als Druck empfunden. Mein Ziel war, nach jeder der neun Lerneinheiten (die letzte war der Leistungsbewertung vorbehalten) eine andere Szene zu drehen. An dieser Stelle stellt sich mir die Frage, ob ich mich zu sehr auf die Zielbeschreibungen 
und auf das Endprodukt der gefilmten Szene konzentriert habe. Ich finde es jedenfalls für mich persönlich befriedigender und motivierender, wenn ich mich auf Sprachlern- und Gruppenprozesse sowie auf Lernerbedürfnisse konzentrieren kann.

Aus rechtlicher Sicht ergibt sich die Problematik der privacy bzw. die des Rechts auf das eigene Bild, denn in diesem Projekt wurden ja Emailadressen und Bilder - und damit private und „sensible“ Informationen - ausgetauscht und in gewisser Weise öffentlich gemacht (zur Umgehung von privacy und zur Kontrolle über die Lernenden in virtuellen Lernumgebungen cf. Swertz \& Dzierzbicka 2008). In einem Nachfolgeprojekt sollte jedenfalls die schriftliche Erlaubnis der Lernenden zur Verwendung ihrer Handyfilme in didaktischen und wissenschaftlichen Kontexten eingeholt werden.

\subsection{Ausblicke}

Eine interessante Weiterführung des vorliegenden Projekts wäre es, die szenischen Interaktionen nicht nur in deutscher Sprache, sondern mehrsprachig auszuführen und so das Konzept der Mehrsprachigkeit dramapädagogisch auszuleuchten. Außerdem wäre es interessant, die Interaktionen zu wirklich dramatischen Interaktionen auszuweiten. Ein weiteres noch zu bearbeitendes Forschungsfeld ist die im Lauf des vorliegenden Artikels angesprochene filmpädagogische Arbeit.

Auch die Nutzung von Handyfilmen zu Evaluationszwecken sowie die damit verbundenen psychologischen Auswirkungen des Sich-selbst-Wieder-Sehens beim Agieren in der Fremdsprache, könnten gerade angesichts der leichten Handhabung von Handyfilmen noch weiter vertieft und erforscht werden.

\subsection{Schlussbemerkung}

Im Grunde versucht mein Projekt eine Möglichkeit aufzuzeigen, kognitive Lernziele (aus dem Referenzrahmen) mit ganzheitlichem lernerorientiertem Lernen (Dramapädagogik) und medienpädagogischen Aspekten (Handyfilm) zu verbinden. Ich hoffe also, dass ich trotz der eben erwähnten Grenzen des Projekts zeigen konnte, wie ich meine dramapädagogische Arbeit in diesem Kurzprojekt einerseits teilweise an die Prinzipien des Referenzrahmens gebunden habe, andererseits aber versucht habe, dessen Standardisierungstendenzen in Individualisierungspotential umzuwandeln. Ich halte die Anbindung an den Referenzrahmen für wichtig, damit sich Dramapädagogik sowohl in den Diskurs der aktuellen fremdsprachlichen Debatte einbindet, als auch die fremdsprachlichen (kognitiven) Ziele nicht aus den Augen verliert. Mein Ziel war und ist es, dass dramapädagogische Elemente noch mehr auch mit Anfängern im curricularen Unterricht einen Platz finden. Gleichzeitig habe ich auch die Grenzen des Referenzrahmens deutlich vor Augen geführt und mit dem Fokus auf Fiktionalisierung und Individualisierung Alternativen aufgezeigt. Der Einsatz des Handyfilms in der Hand der Lernenden bringt, wie ich gezeigt 
habe, zwar zusätzliche Probleme mit sich, die es aber meines Erachtens wert sind, rechtlich, sowie medien- und filmpädagogisch noch weiter durchdacht zu werden. Schließlich ist die Handyfilmkamera ein Medium bzw. hier: ein Lernmedium, mit dem alle Lernenden aufgewachsen sind, und deren Einsatz sicher noch viele andere Möglichkeiten in sich birgt, die es aufzuzeigen gilt.

Die Autorin dankt Diana Feick, Herder-Institut Leipzig, für die Anregung zur möglichen didaktischen Nutzung von Handyfilmen.

\section{Bibliografie}

Anderson, John R. (2001): Kognitive Psychologie. Heidelberg: Spektrum

Bausch Karl Richard / Christ Herbert / Königs Frank / Krumm Hans-Jürgen (Hrsg.) (2003): Der gemeinsame europäische Referenzrahmen für Sprachen in der Diskussion. Tübingen: Narr

Bünger, Ursula Christine (1999): La 'drammapedagogia' nell'insegnamento delle lingue straniere. In: Quaderni del LEND, Milano, dicembre 1999, anno XXVIII 5, 18-24

Eckert, Johannes / Riemer, Claudia (2000): Awareness und Motivation: Noticing als Bindeglied zwischen kognitiven und affektiven Faktoren des Fremdsprachenunterrichts. In: Riemer, Claudia (Hrsg.): Kognitive Aspekte des Lehrens und Lernens von Fremdsprachen. Festschrift für Willis J. Edmondson. Tübingen: Narr. S. 228-246

Europarat (2001): Gemeinsamer europäischer Referenzrahmen für Sprachen lernen, lehren, beurteilen. Berlin u.a.: Langenscheidt

Gass, Susan (1997): Input, Interaction and the second language learner. Mahwah, New Jersey: Lawrence Erlbaum Associates

Kramsch, Claire (1993): Context and Culture in Language Teaching. Oxford: Oxford University Press.

Krashen, Stephen (1985): The input hypothesis: Issues and implications. Harlow, UK: Longman

Krumm, Hans-Jürgen (2008): Gemeinsamer europäischer Referenzrahmen für Sprachen: Ziele, Wirkungen und Nebenwirkungen. In: Fremdsprache Deutsch 38, 26-28

Krumm, Hans-Jürgen (2006): Müssen jetzt alle dasselbe können? Vor- und Nachteile der Globalisierungsprozesse im Sprachunterricht. In: Fremdsprache Deutsch 34, 30-33

Krumm, Hans-Jürgen (2001): Die sprachlichen Fertigkeiten: isoliert kombiniert - integriert. In: Fremdsprache Deutsch 24, 5-12

Lave, Jean / Wenger, Etienne (1991): Situated learning: Legitimate peripheral participation. New York: Cambridge University Press

Martinez, Helene (2005): Lernerautonomie: ein konzeptuelles Rahmenmodell für den Fremdsprachenunterricht. .. und für die Fremdsprachenlehr- und lernforschung. In: Fremdsprachen lehren und lernen 34, 65-82 
McLuhan, Marshall (1964): Understanding Media. The extensions of Man. New York: McGraw-Hill

Neuner, Gerhard / Krüger, Michael / Grewer, Ulrich (1981): Übungstypologie zum kommunikativen Deutschunterricht. Berlin u.a.: Langenscheidt

Schewe, Manfred (1993): Fremdsprache inszenieren. Zur Fundierung einer dramapädagogischen Lehr- und Lernpraxis. Oldenburg: Zentrum für pädagogische Berufspraxis der Carl von Ossietzky Universität Oldenburg

Schmenk, Barbara (2004): Drama in the Margins? The Common European Framework of Reference and its Implications for Drama Pedagogy in the Foreign Language Classroom. In: German as a Foreign Language (GFL-Journal) 1, 7-23 http://www.gfl-journal.de/1-2004/schmenk.pdf (30/01/2011)

Schmidt, Richard (1990): The role of consciousness in second language learning. In: Applied Linguistics 11, 129-158

Swain, Merrill (1985): Communicative Competence: Some Roles of Comprehensible Input and Comprehensible Output in its Developement. In: Gass, Susan M. / Madden, Carolyn (Hrsg.): Input in Second Language Acquisition. Rowley, Mass.: Newbury House, 235-253

Swertz, Christian / Dzierzbicka, Agnieszka (2008): The decomposition of privacy. The shift of power in virtual learning rooms. In: Kommer, Piet; Isaias, Pedro (Hrsg.): e-society '08. Proceedings of the IADIS International Conference, 597-601

Wettemann, Ulrich (2007): Kein trockener Anfängerunterricht mehr! Eine dramapädagogische Modelleinheit für die ersten DaF-Stunden. In: Scenario (1) http://publish.ucc.ie/scenario/2007/01/wettemann/04/de (30/01/2011) 


\section{Anhang}

\section{Im Café}

Guten Tag. Bist du neu hier?Ja.Wie heißt du? Mein Name ist Jessica.Wie alt bist du? Ich bin 17 Jahre alt.Wo wohnst du? Ich wohne in Ragusa.Woher kommst du? Ich komme aus Italien. Was ist dein Hobby?Mein Hobby ist Chatten.Toll.

\section{Am Kaffeeautomaten}

Guten Tag. Bist du neu hier?Ja.Was willst du? Ich will eine warme Schokolade. Und du? Ich will einen frischen Orangensaft. Wie ist deine Handynummer? Meine Handynummer ist ........ Das ist richtig so?Ja genau!Wie heißt du? Ich bin Irene, und wie ist deine Handynummer? Meine Handynummer ist ....Und wie heißt du? Ich bin Maria!Bis bald!

\section{Auf der Schulhofmauer / In der Arztpraxis}

Willst du eine Zigarette?Nein danke.Lernst du Englisch?Jan natürlich lerne ich Englisch. Und du?Ich auch.Sprichst du gut Englisch?Ja, ich spreche Englisch gut.Ich mag Englisch. Magst du Englisch?Ja, ich mag Englisch. Wie heißt deine Englischlehrerin?X und deine?Y. Was ist denn dein Lieblingsfach?Mein Lieblingsfach ist Spanisch. Und deins?Mein Lieblingsfach ist Englisch.Ach so. Ich muss in die Klasse. Tschüss!Ich auch. Tschüss!

\section{Auf der Polizeiwache}

Haben Sie eine Schwester?Ja ich habe eine Schwester.Wie heißt sie?Sie heißt Stefania.Wie alt ist sie?Sie ist 24 Jahre alt.Haben Sie ein Tier?Ich habe ein Kaninchen.Es war das Kaninchen!Nein!

1. Im Beduinenzelt

Hast du ein Taschentuch?Nimm!Mir ist heiß.Du kannst warmen Tee trinken.Das will ich nicht.Du kannst Eis essen.Nein, danke. Ich will eine kalte Cola.

1. Bei der NASA

Hast du einen Bleistift?Nimm!Danke. Wie komme ich zum Mond?Du kannst mit dem Fahrrad zum Mond fliegen.Wie komme ich zur Sonne?Du kannst mit der U-Bahn zur Sonne fahren.Ich will zum Mars.Kein Geld! 